\title{
Survey of the use of data in UK bridge asset management
}

John Bennetts MA, MSc, EngD, GMICE

Senior Bridge Engineer, WSP, Bristol, UK

Paul J. Vardanega BE, MEngSc, PhD, GMICE, MASCE, MIEAust, FHEA

Senior Lecturer in Civil Engineering, Department of Civil Engineering,

University of Bristol, Bristol, UK (corresponding author:

p.j.vardanega@bristol.ac.uk) (Orcid:0000-0001-7177-7851)
Colin A. Taylor BSc, PhD, CEng, FICE

Emeritus Professor of Earthquake Engineering, Department of Civil Engineering, University of Bristol, Bristol, UK

Steve R. Denton MA, PhD, CEng, FREng, FICE, FIStructE Head of Civil, Bridge and Ground Engineering, WSP, Bristol, UK

Considerable amounts of data are collected on the UK's stock of bridges. Much of this data is collected to inform the planning and scope of maintenance activities. This paper reports on the results of a series of semi-structured interviews with 17 individuals involved in UK bridge asset management and data-collection activities to explore how such data is used in practice. A wide spectrum of organisations and industrial sectors was represented in this dataset. Hierarchical process modelling was used to characterise the UK's bridge management system and define the processes and sub-processes involved in the management of bridges. Key quotations are used from the interviews to reveal the state of data collection and use in UK bridge infrastructure from the perspective of those directly involved. The study concludes that there is significant variation within the industry regarding the use of visual inspection data and that formal structural health monitoring remains relatively rare. Furthermore, there is a need to develop a new unifying paradigm that will frame the efficient and effective application of emerging artificial intelligence and data science enabled (i.e. 'smart') condition-monitoring techniques to bridge management.

\section{Introduction}

The use of data in bridge management organisations is an important topic as the civil engineering profession seeks to understand the potential impacts and challenges of emerging artificial intelligence and data science enabled ('smart') condition-monitoring techniques. The UK has an ageing stock of infrastructure, which could be referred to as an 'asset time bomb' (Thurlby, 2013). Making better use of the collected data on bridge condition may assist with better management and maintenance of a nation's bridge stock. The systems and processes by which data is used within bridge management, especially when planning maintenance interventions, are often not clearly reported in the literature because, usually, these processes are unique and internal to specific organisations. This paper aims to use information obtained from a series of semistructured interviews with a representative set of individuals involved in bridge management at various organisational levels to 'map' the system of bridge management, understand what data is collected and how it is used. This information is then used to gauge how well prepared the UK bridge management sector is for the emergence of smart condition-monitoring techniques.

Preliminary findings from this study were reported by Bennetts et al. (2016). This paper gives the full set of results from the study. The findings are presented in two forms

- key quotations from a series of semi-structured interviews (explained in more detail in Section 2.1)
- hierarchical process modelling (HPM) to capture what goes on and why with bridge management in the UK (explained in more detail in Section 2.2).

Further details on the 'Management of bridges project' are provided in the thesis of Bennetts (2019), which also includes some of the work reported in this paper.

\section{Methodology}

\subsection{Semi-structured interviews}

This study involved the use of semi-structured interviews with people engaged in bridge management activities in the UK. In such a study, the interviewees should be representative of UK bridge owners and managers (i.e. the population) (e.g. Oppenheim, 1992). Therefore, those interviewed were chosen to be representative of those involved or who have a professional interest in UK bridge management. The interviewed group included policy setters in major organisations, inspectors and managers of individual assets. All modes of transport (e.g. rail and highways), the level of organisational remit (i.e. strategic, city region and local authority) and links in the supply chain were sampled. In total, 14 interviews were conducted, with 17 participants. The organisational roles and sectors of those interviewed are presented in Table 1. Throughout this paper, quotations from those interviewed are presented and referenced using the notation shown in Table 1 (e.g. C1) in brackets following the quotation. For brevity, the 
Table 1. Details of the interviewees' roles and sectors (adapted from Bennetts et al. (2016))

\begin{tabular}{|c|c|c|c|c|}
\hline & Interview & Role & Sector & Scope \\
\hline $\mathrm{C} 1$ & 1 & Senior policy advisor & Highways & Strategic \\
\hline $\mathrm{C} 2$ & 2 & Structures manager & Highways & Metropolitan transport authority \\
\hline C3 & 3 & Structures manager & Highways & Local \\
\hline C4 & 3 & Structures engineer & Highways & Local \\
\hline C5 & 4 & Structures asset manager & Rail & Strategic \\
\hline C6 & 5 & Structures manager & Rapid transit & Metropolitan transport authority \\
\hline $\mathrm{C7}$ & 6 & Regional structures specialist & Highways & Strategic \\
\hline $\mathrm{C} 8$ & 7 & Head of engineering & Highways & Strategic, concessionaire \\
\hline C9 & 7 & Assistant head of engineering & Highways & Strategic, concessionaire \\
\hline C10 & 8 & Researcher & Highways & Local, heritage \\
\hline C11 & 9 & Structures watchman & Highways & Strategic, service provider \\
\hline $\mathrm{C} 12$ & 10 & Chief bridge engineer & Rail & Strategic \\
\hline $\mathrm{C} 13$ & 11 & Professor & Academia & National \\
\hline C14 & 12 & Head of bridge engineering & Engineering consultant & International \\
\hline C15 & 13 & Chief executive officer & Highways concessionaire & Strategic \\
\hline C16 & 13 & Technical director & Highways concessionaire & Strategic \\
\hline C17 & 14 & Bridge specialist & Highways & Strategic \\
\hline
\end{tabular}

paper is necessarily selective. The transcripts of the interviews cannot be released.

Standardisation of the interviews was achieved by using a common interview protocol to explore the important research topics and areas of interest for the investigation. All the interviews were performed by the first author, with care taken to avoid leading the interview candidates. The research consciously adopted a 'mixed methods' approach, combining hypothesis-led positivist-style questions such as 'what data do you collect on your structures?' (from which a closed-form answer is expected) with response-led lines of questioning and discussion, where the interview protocol was used to define the theme of the discussion rather than the precise questions used. This latter style of questioning for themes such as 'how does your organisation make decisions?' represents a grounded theory approach (Glaser and Strauss, 1967). The interview protocol is provided in Table 2.

The interviews were recorded digitally and then analysed by 'coding' against research questions and emerging themes in the audio files (e.g. Saunders et al., 2009). This 'coding' of interview transcripts or audio files consists of highlighting snippets of the interview that relate to each of the research questions or emerging themes identified during analysis of the transcripts. A series of codes (Table 3) were set up and these were then applied to relevant sections of the interview audio files. The coding schema (Table 3) was developed inductively and updated as the interviews were processed. This resulted in an iterative process as already coded files were reviewed and reflected upon as new files were analysed and themes emerged. Traditionally, these approaches would have been applied manually with page markers and highlighters in the transcripts.
Table 2. Interview protocol (running order indicated)

Interview question/theme
Introduction
Q1 What is your role?
Q2 What does your role involve on a day to day basis?
Q3 How long have you been in your current role?
Collection of asset data
Q4 What data do you collect on your bridge assets?
Q5 How do you collect this data?
Use of asset data
Q6 How do you use the data that you collect on your
Q7 Are there any IT systems that you use to manage and
The decision-making process
Q8 How do you make decisions regarding the management
The overall process of managing bridges
Q9 Exercise completed on paper: HPM
Open question
Q10 Looking at the model we have produced, which aspects
$\quad$ of your role keep you up at night?

In this work, Dedoose.com (a computer-aided qualitative data analysis software package) was used to transcribe the audio and apply the codes as a digital pack of highlighters and bookmarks in the transcript files. This software is able to maintain a link to the underlying interview recording in-line with the transcription, so that when it came to reviewing the emerging themes and conclusions, the relevant snippets from each of the interviews could be viewed alongside each other and played to check the context and tone of the quotes before inclusion in the narrative. By enabling each relevant extract to be reviewed alongside others, the software helped to reduce bias between 
Table 3. Coding schema applied to the interview data

\begin{tabular}{ll} 
Ref. & Code \\
\hline 00 & Great quotes \\
1 & Data collected on assets \\
1.1 & Monitoring \\
1.1 .1 & SHM \\
1.2 & Visual inspection \\
1.2 .1 & Risk-based inspection intervals \\
1.2 .1 & Reliability of visual inspection \\
1.3 & Inventory data \\
1.4 & Maintenance actions \\
1.5 & Prioritisation of inspection effort \\
2 & How is data collected \\
3 & How is data recorded \\
3.1 & Database \\
4 & IT systems used \\
4.1 & Recent or upcoming changes to IT systems \\
5 & How data is used \\
5.1 & Identifying need \\
5.2 & Trends in data \\
5.3 & Future aspirations \\
5.4 & Tool for measuring service provider performance \\
5.5 & Audit trail \\
5.6 & Producing condition scores \\
5.7 & Model validation \\
5.8 & Targeting further testing/inspection \\
5.9 & Informing assessment \\
6 & How decisions are made \\
6.1 & Judgement \\
6.2 & Deterioration modelling \\
6.3 & Value for money \\
6.4 & Standard maintenance periods/plans \\
6.5 & For assessment work? \\
6.6 & Assumptions on lifespan? \\
6.7 & Prioritisation \\
6.8 & Asset lifecycle planning \\
6.9 & Whole-life cost \\
6.10 & Peer review \\
6.11 & Heuristics \\
7 & HPM exercise \\
&
\end{tabular}

the interviews and allowed auditing of the key results among the authors. All the interview extracts have been carefully anonymised. In some cases, the quotations were slightly altered to improve the grammar but the original meaning was preserved.

\subsection{Hierarchical process modelling}

'Complicated processes' can be reduced to a hierarchy of increasingly simple sub-processes (Hall et al., 2004). In the hierarchical tree structure, 'child processes' answer the question 'how do we do this process?' and 'parent processes' answer the question 'why do we do this process?' This type of modelling by individual 'experts' has been applied in various attempts to model the performance of civil engineering systems with a significant degree of complexity and uncertainty (e.g. Blockley and Godfrey, 2000; Hall et al., 2004).
In many examples (e.g. Blockley et al., 2012; Davis and Hall, 2003), this technique is combined with Italian flag notation and interval probability theory to quantify the uncertainty in decision-making systems. However, it is stressed that the models presented in this paper are ontological rather than mathematical/computational - that is, the word 'model' refers to a conceptual model of the interconnected processes of bridge management in the UK to aid understanding of the way in which processes interlink. The novelty of this work lies in the interview results and the topography of the hierarchical process diagrams, not in the further development or justification of HPM approaches.

HPM is a method of eliciting and formalising 'expert' knowledge and heuristics. However, it is not anticipated that any single 'expert' would be able to produce a model of the UK's bridge management system encompassing all its facets because there are many different agents within the system, each of which has different visibility and perspectives on the whole system. Davis et al. (2010) reported the successful use of group sessions for building hierarchical process models when 'no single person can understand all aspects, issues and variables of such an intricate problem'. Lane (1992) argued that the approach of individuals building conceptual systems models as 'experts' can reduce stakeholder trust in the models and the chances of making successful interventions. Flaig and Lark (2000) published a study of bridge management systems in the UK after conducting an extensive set of interviews and concluded that '... current systems are often too theoretical in the approach they take to the practical task of managing a bridge stock...' (Flaig and Lark, 2000; p. 105). Reviewing the options for building models of the bridge management system, it was therefore considered preferable to hold group modelling sessions with stakeholders from different organisations in the system. However, in this case, because of the organisational and spatial distribution of the key stakeholders, it was not viable to convene a meaningful panel to engage in a group modelling exercise. Therefore, the process of group model building was distributed across a series of smaller, often individual, facilitated model building sessions. In this way, individual sessions were held with key actors to produce a model that captured their perspective on the overall system. These individual models were then synthesised by the authors into one over-arching model that encompassed all the individual perspectives. The modelling sessions were introduced as part of the semi-structured interviews, with candidates introduced to an example of a simple hierarchical process model for 'being a water plc' and 'owning a dam', as presented by Blockley et al. (2012). The interviewees were then given a sheet of paper printed with the starting point for a bridge management model, as shown in Figure 1. This 'seed' was used a starting point and to set the context and scope for the interviewee's diagrams. These seeds were adjusted to reflect the sector and scope of each candidate's role. 


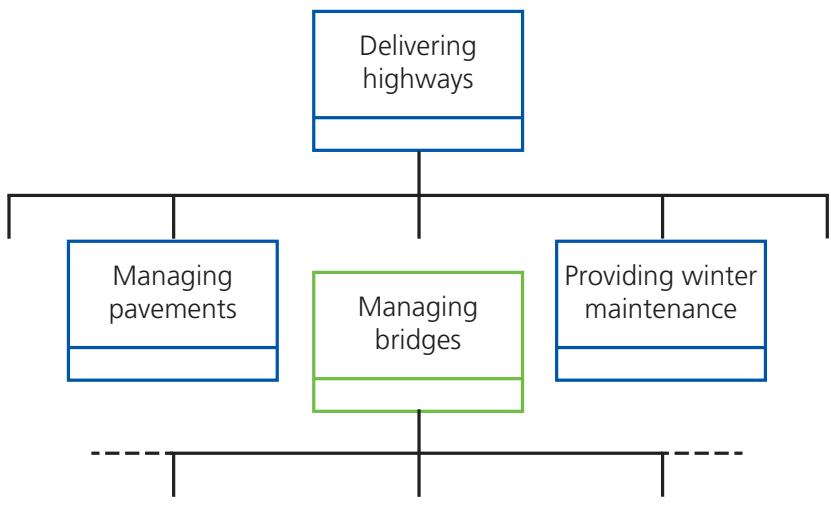

Figure 1. Seed (example) hierarchical process model presented to the candidates in the highways sector

\section{The bridge management process: modelling}

At the top level, all of the processes identified by candidates could be categorised under three fundamental processes

understanding the stock

- making decisions

implementing interventions.

These fundamental processes operate within a framework that is defined by the organisation's higher-level objectives and industry codes of practice and standards, and is bounded by budgetary constraints. Figure 2 shows the three identified core

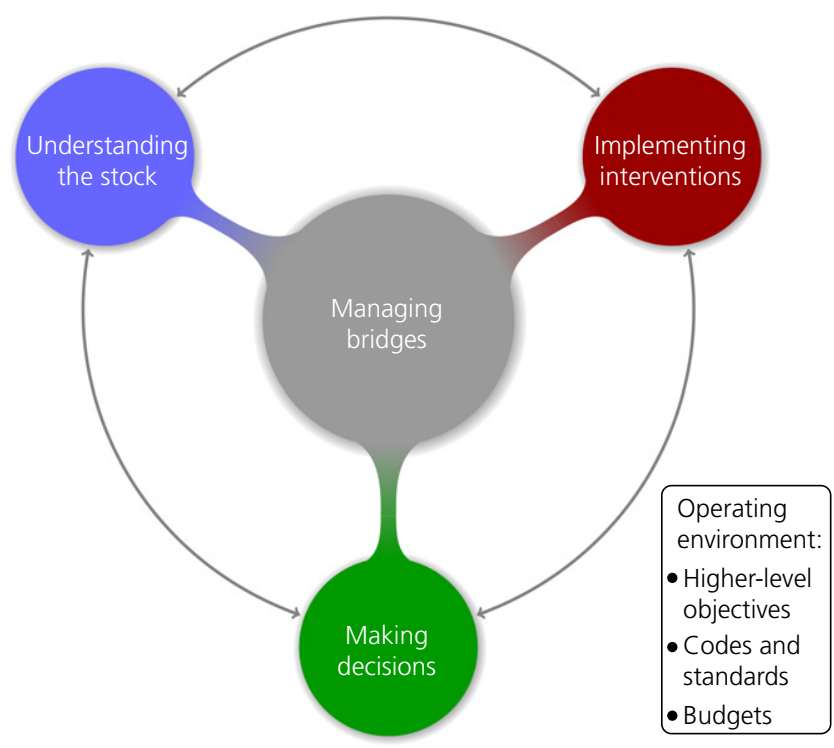

Figure 2. High-level model of the bridge management system processes, which often follow sequentially (anti-clockwise in this diagram), and cyclically, in time and therefore could also be taken to represent a 'traditional plan-act-observereflect cycle' (Kemmis et al. 2014: p54).

For strategic-level organisations, significant links and interactions were noted between the processes within the bridge management system and the operating environment, with some seeking to control the operating environment by justifying increases to budget, contributing to standards and informing objectives. In effect, these organisations have been able to expand their system boundary and engage in the national socio-political system to improve their position. For some organisations, a significant portion of the interviewed individual's efforts were reported to be dedicated to the creation and maintenance of relevant codes and standards. However, this was considered to be an activity outside the core process of managing bridges. Sections 4-6 give some selected key quotations from the semi-structured interviews that fall under the three identified top-level processes of the HPM: implementing interventions, understanding the stock and making decisions.

\section{Uses of bridge asset data: implementing interventions}

Bridge managers have a relatively small number of fundamental intervention options open to them. They may (a) implement maintenance interventions to existing bridges to renew their condition, (b) improve existing structures to increase their carrying capacity (either in lane width or load rating) or (b) demolish or replace structures at the end of their service lives.

Most bridge owners reported programmes of routine maintenance, and systems for reacting quickly to urgent safety-critical issues reported through inspections, user hot-lines or network control centres. Another key activity was reported to be maintaining an engaged and proactive supply chain, which was noted to be crucial to enabling cost effective interventions: 'if you don't get the [implementation of interventions right] everything else will be a waste of time because you need a supply chain that is with you and like you' (C15). Figure 3 shows the developed HPM for 'implementing interventions'. Implementing interventions can be broken down into: maintaining structures, improving structures, replacing structures and managing supply chains.

\section{Uses of bridge asset data: understanding the stock}

Maintaining a deep and up-to-date understanding of the stock of bridges an organisation is responsible for is crucial to being able to plan activities and expenditure effectively to manage the risks caused by ageing infrastructure, environmental 


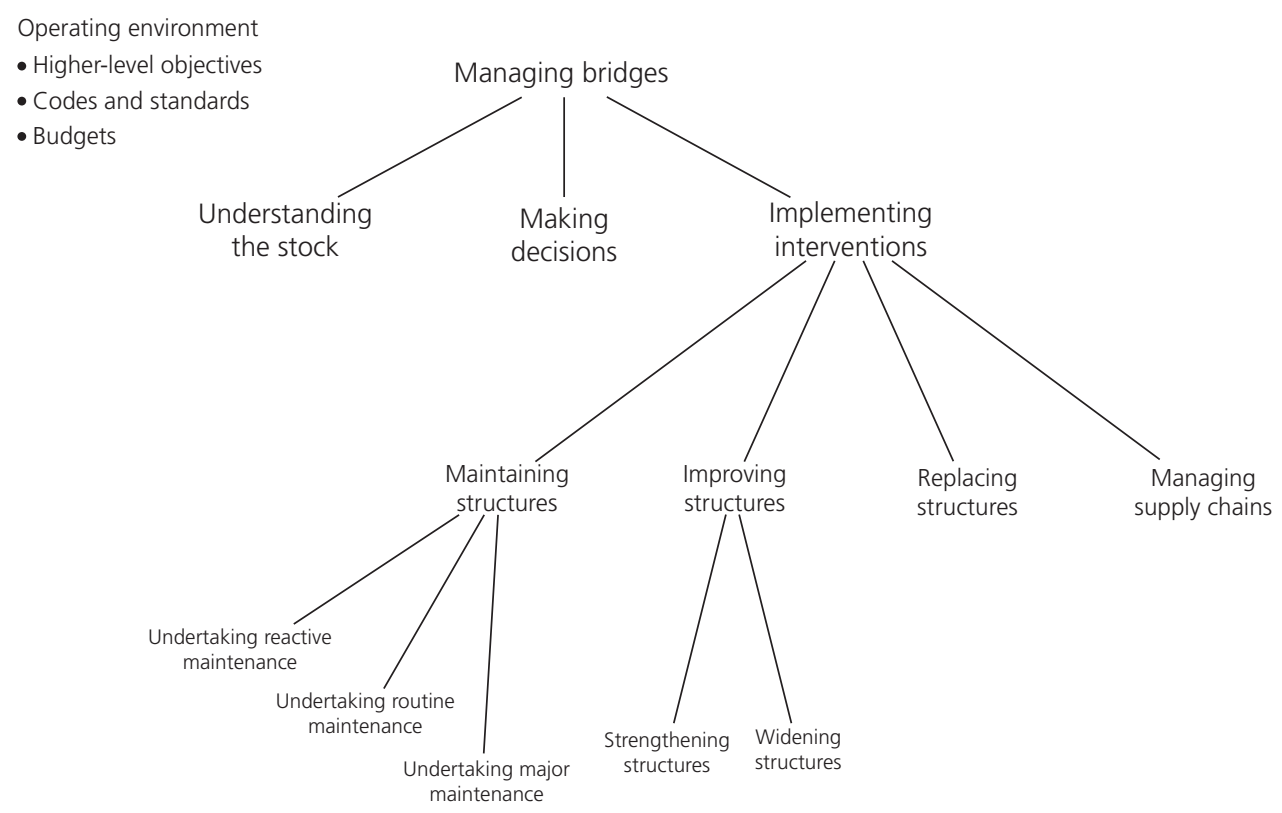

Figure 3. Hierarchical process model of the implementing interventions process within the high-level process of managing bridges

hazards and accidental events. Maintaining this understanding comprises four key activities

compiling and maintaining an inventory of the structures an organisation is responsible for

monitoring and maintaining records of their condition

- maintaining records of their capacity

- managing risks to safety or functionality.

Figure 4 shows the branch of the developed HPM for 'understanding the stock', from which four primary nodes were identified: compiling inventory, understanding condition, understanding capacity and understanding risk. Some key quotes related to understanding the stock are now presented.

\subsection{Compiling inventory and recording data}

The majority of interviewees reported that their bridge information is held in dedicated databases that typically hold inventory, inspection and maintenance data. These databases often also hold the results of load-rating assessments and risk assessments such as for scour or road safety. The maturity of these tools varies, with a few organisations relying on spreadsheets for some aspects of their data management, while others have complex integrated IT solutions. Many participants mentioned either newly implemented or imminent IT solutions: 'we're in the process of rolling it [the new system] out ... it pulls all those databases together, so we've got one version of truth' (C5). On the development of a new system, another interviewee stated 'well it's still in its infancy, I mean we've probably been running it for 3 or 4 years now and it's evolved slightly as well ... we've now got a refined approach ... we'll refine the process as well and keep reviewing it, and it'll become better and better and also we'll have more historical data to be able to verify against as well' (C2).

\subsection{Visual inspections}

Asset managers need a rational approach to allocate maintenance and upgrade resources. As a result, visual inspections are carried out to assess the level of deterioration of bridge assets (typically principal inspections, which involve up-close inspection of all elements to within touching distance performed at intervals of 6 years, but may the interval may be adjusted on a risk basis (HE, 2017; NR, 2017)). Visual inspections are reliant on the interpretation (and judgement) of the bridge inspector on site and are carried out often in difficult conditions such as within confined spaces with poor lighting. Studies have shown that there is variability in the scores given by different inspectors during visual inspections and therefore the data itself suffers from inevitable fuzziness and variability (e.g. Graybeal et al., 2002; Lea and Middleton, 2002; Middleton, 2004; Moore et al., 2001). Bennetts et al. (2018a, 2018b) demonstrated with more recent data that, while variability in scores is evident for individual defects, visual inspection can capture deterioration rates at stock level.

All the represented organisations in the survey set make use of visual inspections as the primary condition data source. Many organisations appear to see it as a key driver of the 


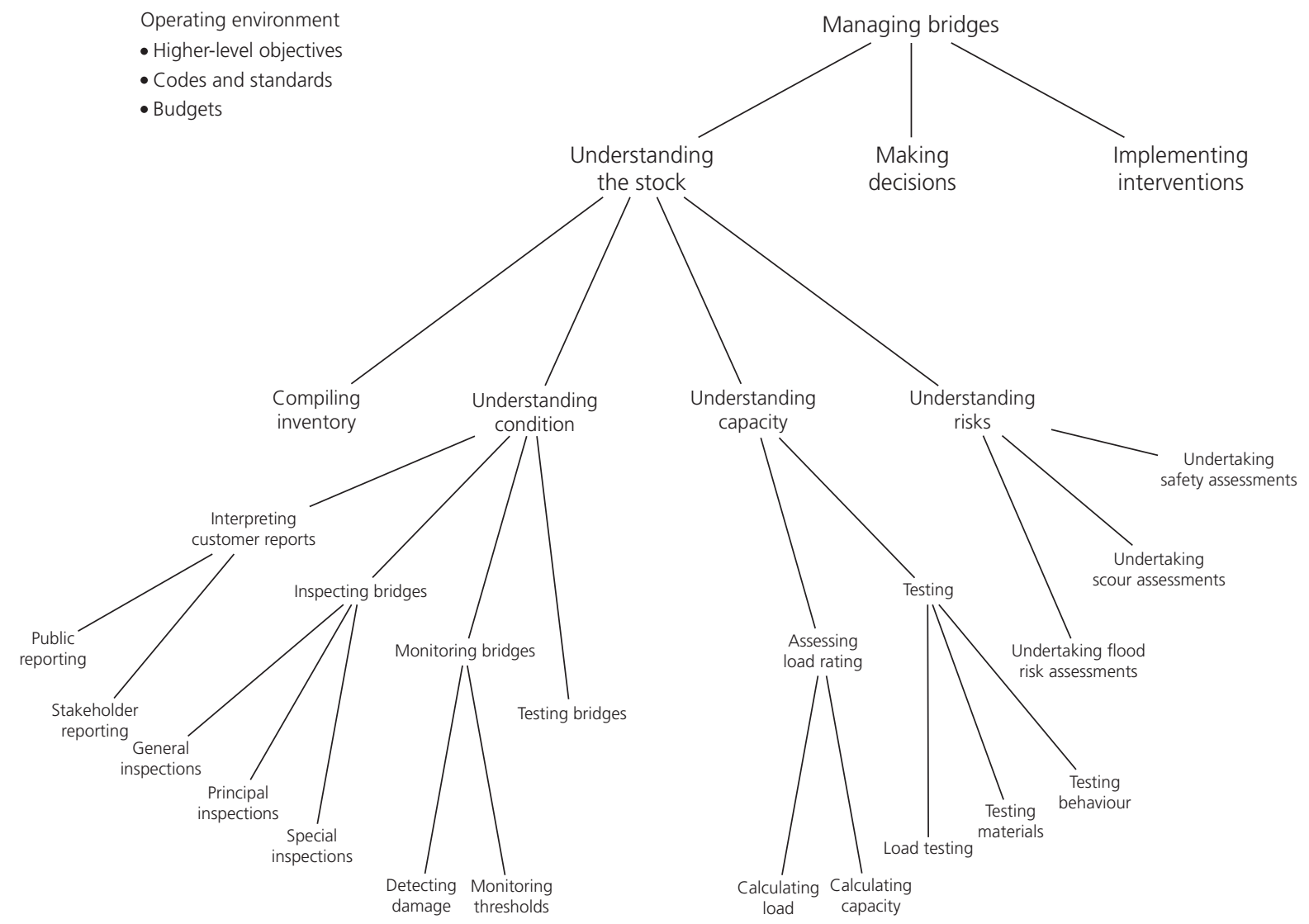

Figure 4. Hierarchical process model of the understanding the stock process within the high-level process of managing bridges

management of their structural assets. One interviewee stated 'inspections are, really, the foundation for everything we do' (C3). Most inspection recording protocols record condition data as the nature, severity and extent of the defects, mostly using the County Surveyors' Society system described in HA (2007), or adaptations thereof. While using a similar approach, the rail sector records defect risk in terms of consequence and likelihood. Visual inspections are also used to record maintenance activities that may be linked to specific structural defects and the indicative costs allocated: 'we record suggested remedial works, indicative prices, that sort of thing' (C4). Recently, many organisations have begun to extend the inspection intervals for some structures beyond 6 years on a risk basis: 'the cycle is dependent on risk, so if you've got a brand new concrete or weathering steel structure you might want to look at it less frequently' (C5). One interviewee questioned if the frequent general inspections, which are more superficial in nature, offer value in the current inspection regime. 'Take a general inspection, I half think you're doing it for your own self-conscious, I don't think there's much merit in it' (C15). Interestingly, the rail sector's programme of inspections has been aligned with necessary inspections for an 18-year cycle of steady-state load-rating assessments so that 'every 18 years you will get an engineer doing an examination [whereas otherwise] ... our examiners are generally ex-trades[people]' (C5). The need to ensure reliability of collected inspection data was noted by some interviewees: '... subsequently we obviously make the decisions on it, and if you're making it on the basis of unreliable data then that's clearly poor practice' (C1). Some interviewees noted that they had undertaken informal comparisons of the inspection results from different inspectors and found significant variation. Some interviewees discussed a lack of confidence in the quality of inspections delivered by their suppliers: 'we are finding the quality of those inspections that we're getting done externally is ... inadequate' (C2). Some participants are examining the possibility of changing how their inspections are delivered: 'it may be that inspections are handled in-house or maybe with a contract that's separate from our [highway maintenance] service providers' $(\mathrm{C} 1)$.

Looking to the future, the participants disagreed on the role of visual inspections to maintain an understanding of bridge condition. Some saw that there would always be a role for visual inspections, perhaps augmented with technology '... visual 
inspection will still form the basis of most inspections, quite rightly, with competent trained individuals, but using photographs and video to get an objective image which is then automatically overlain on the existing model, and tracks changes with time' (C13). Others foresee a time when a combination of technologies would be used to monitor a bridge's condition and predict its future, with one interviewee (C15) stating

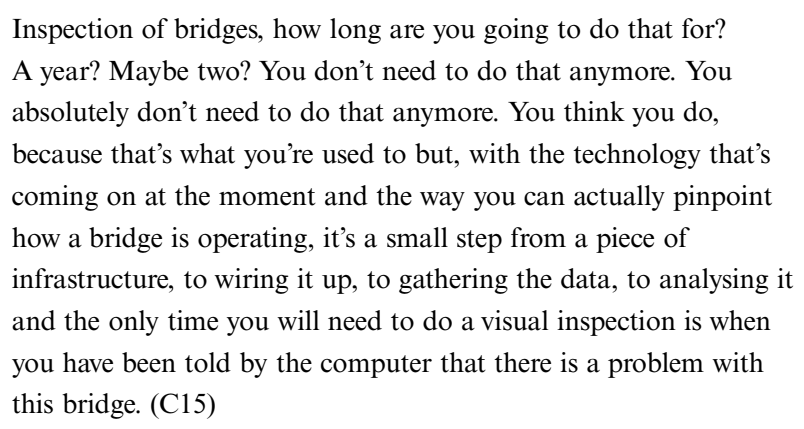

The authors note that while 'damage detection' such as this is a highly valuable aim of monitoring systems, it is difficult to reliably achieve in practice (cf. Webb et al., 2015).

\subsection{Monitoring inspections}

In some situations a structural element will require enhanced data collection (e.g. higher frequency or improved fidelity of data) than that collected during routine visual inspection processes. In such cases, most of the represented organisations would implement a programme of 'monitoring inspections'. The inspection periods would then be reviewed depending on the defect severity, deterioration (ongoing) and the element's importance in the asset: 'it's a balance between keeping everything safe, and keeping an eye on everything and working within the resources we're given' (C3).

\subsection{Structural health monitoring (SHM)}

One interviewee $(\mathrm{C} 13)$ pointed out

\begin{abstract}
The vision we talk about is of a ubiquitous world where, like with the sensors in modern cars, the bridges tell us everything about their performance. I think we've got quite a long way to go to get to that, but it should be an ambition; however, whether it is a viable and economic vision is still an interesting question. (C13)
\end{abstract}

More recent efforts to put monitoring as central to infrastructure asset management (cf. Davila Delgado et al., 2017; Middleton et al., 2016) mean that many researchers argue that new SHM technologies (e.g. Hoult et al. 2009) may in the future supplant visual inspection as the main type of SHM (cf. McRobbie et al., 2015). SHM systems offer the prospect of reduced levels of traffic disruption as well as the potential for more frequent data-collection points and nodes. The use of SHM systems appears to be limited to instances where a specific structure, critical to the network operation, has a particularly serious defect (e.g. the case of the Hammersmith flyover (Webb et al., 2014)): 'we have specific monitoring, so if we've got a specific problem we're concerned about and we want to gain information about it then we will ... have targeted monitoring, [that] definitely will help with what we need to do... we're talking about a handful of cases' (C2). Similarly, another interviewee stated 'we have, probably, a dozen sites where we have real-time monitoring. They're the stuff we're really worried about... it's not very often, but we do do it' (C5). While another said that if they were to deploy SHM 'it would be, very much, targeted' (C1). One interviewee noted the reassurance that monitoring a structure had given them to keep a structure in service: 'given the choice of doing it again, or not doing it, I would definitely implement it again ... for the peace of mind, and really we needed it for BD 79 - we needed some justification to keep the road open' (C16). Some participants reported that they have 'none [monitoring systems] at the moment... not any remote monitoring' (C11). However, asset managers with large, strategically important assets to manage use SHM: 'Where do we start? We're monitoring wire breaks ... there's wind speed for bridge closure... there's the weigh-in-motion system ...' (C8, C9). Some of those interviewed indicated that, in the future, they could be interested in using SHM: 'I am aware of ... remote monitoring as well' (C3). Another participant stated 'we probably don't do as much as we should' (C2). Others - when asked if there is monitoring they would like to do but currently do not - noted that the condition of their structures does not currently warrant the use of monitoring systems: 'we've not really got anything that is of a serious concern to say I really want that minute-by-minute' (C6). Others noted the cost of monitoring systems as a deterrent: 'part of it would be cost, so can we justify putting it in?' (C2) and looked forward to lower-cost commoditised sensors: 'wouldn't it be good to have a 21 st century inspectors toolbox, ... a box of various cheap widely available and easy to connect sensors?' (C12). Another interviewee noted that 'the use of the data's the key thing, and what I found is when it came to the assessment stage, the use of this data was very poor' (C13). In specifying systems, managers need to ask themselves 'what is this monitoring really going to tell you?' (C5).

Several participants anticipated an increase in the use of image processing techniques to augment and replace more traditional monitoring and inspection methods: 'without question augmented reality and virtual reality are going to be absolute game changers; computer vision is a no-brainer' (C13). One participant noted positive results from replacing traditional strain gauges with digital image correlation: 'we've been doing a combination of strain gauging and digital image correlation to take multiple strain fields over difficult to access areas such as over the railway line. That's a technique we're using more and more, actually' (C14). 


\subsection{Load-rating assessments}

Some participants identified a link between structural condition and assessment of structural capacity

There's interaction between the two sides, so it may be that an assessment triggers an additional inspection. Examination may trigger assessment [which is] more likely than assessment triggering an examination. (C5)

Another interviewee suggested that change in condition can trigger a reassessment of load-rating: 'so it's as things change, or we're aware of some deterioration that effects the assessment, then we look at reassessing' (C3). Verification of the results of structural analysis is another use of monitoring data (i.e. model validation (Webb et al., 2015)): 'as part of the assessment process, we do use strain gauges or whatever, so we can back analyse' (C5).

\section{Uses of bridge asset data: making decisions}

The linkage between recorded data and its use in informing management decisions was noted by some participants in the study: '[the database] is just a repository for data, and perhaps some information, the knowledge is how you use it, and the wisdom is implementing that' $(\mathrm{C} 1)$.

Figure 5 shows the branch of the developed HPM for 'making decisions', again with four primary nodes represented: understanding need, identifying interventions, prioritising work and planning interventions.

\subsection{Identifying and prioritising need}

The most commonly reported use of bridge condition data is to determine the need for maintenance interventions: 'so we get a great big long list [element by element, across all structures], so we can look at that and say those are the sorts of things we need to be looking at, and that's a first pass' (C2). One interviewee reported that they rely on contractors to identify renewals: 'a lot of it relies on our service providers ... to identify need' (C1). Monitoring data is also used to identify needs and target interventions to resolve them

Take the example of acoustic emissions - we collate the data so we know where the highest instances of wire breaks is ... if we did get

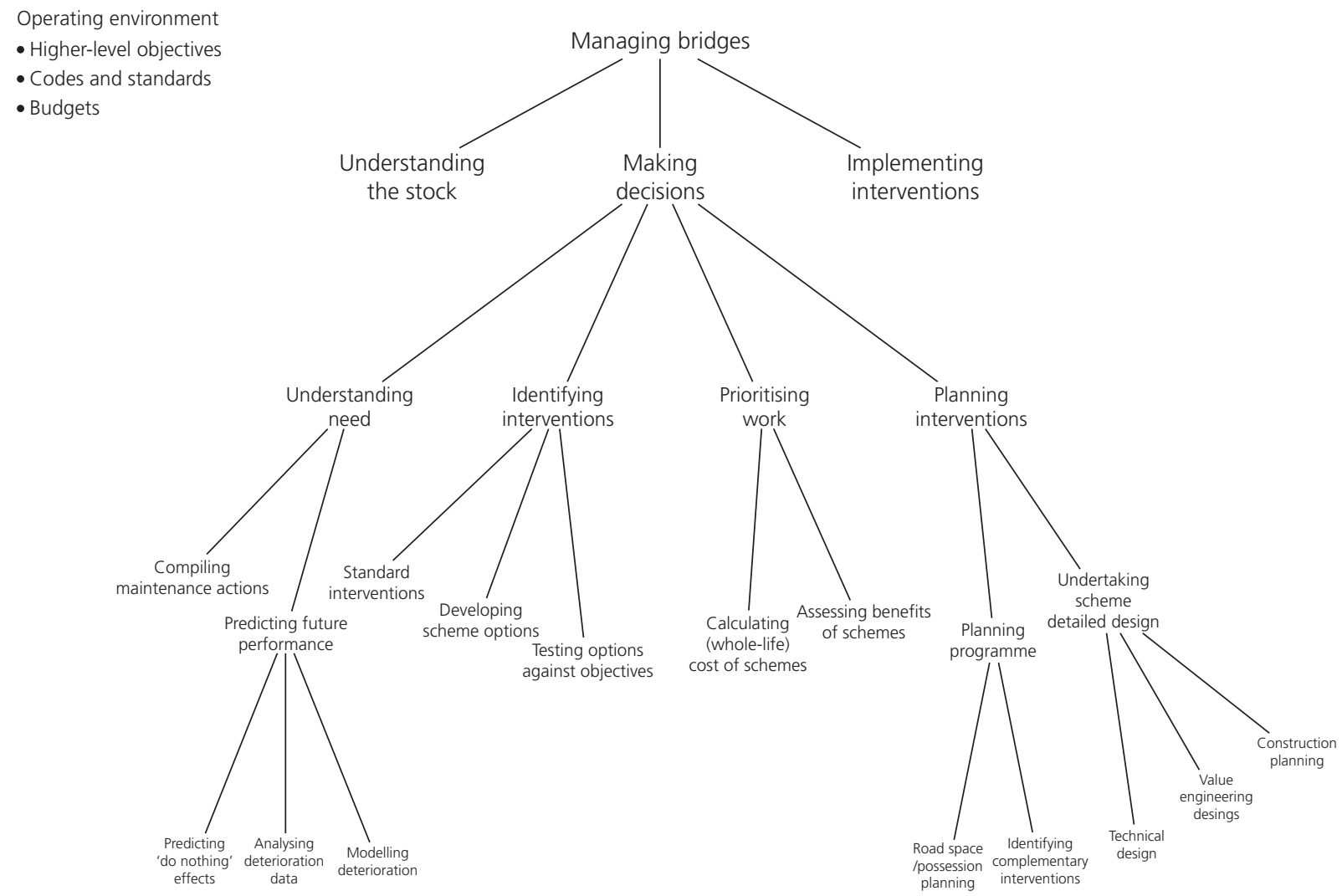

Figure 5. Hierarchical process model of the making decisions process within the high-level process of managing bridges 
a cluster of wire breaks, then obviously when we went in to do our next intrusive inspection, then that [data] would feed into the selection of the panels for the intrusive inspection. (C9)

\subsection{Analysing trends}

The asset owners expressed a desire to monitor trends in subgroups of their stocks: "we look at trends at a family level, so for this family of structures we have the probability of a structure falling from one condition grade to the next' (C12). The ability to analyse trends may be an area for innovation, with one interviewee $(\mathrm{C} 1)$ noting

So we look at trends in condition ... but it's mainly used at a strategic level and obviously what we want to do is to be able to look at trends at an operational level as well ... looking to the future, there's a lot more opportunity to use the data in much smarter ways ... we're not probably very good at looking at trends, so it relies on individual's judgement to say whether we've got problems with particular types of structures. (C1)

\subsection{Maintaining an audit trail}

Another use of the collected data is to provide evidence to justify what work to do and that work needs to be done: 'we have a finite resource; it's about justifying where's the best place to spend it' (C2). Another interviewee explained that the data can also be important in justifying why sometimes work is not carried out: 'that priority score also helps us defend not doing something to politicians or the public' (C3).

\subsection{As a contractual tool}

In some cases, an asset owner will delegate some management decisions to contractors who are charged with maintaining the asset for a given timeframe. Therefore, asset owners have an interest in ensuring that the long-term performance of structures is engendered by the decision-making process. Representatives from two organisations explained that such contractual terms are related to the condition of the assets: 'we have to hand it back in a condition which allows it to be operated for the remainder of its design life' (C9). One interviewee noted contractual terms that specifically mention condition data: 'on a fixed date at the end of the contract they have to hand back all structures with a BCI score of 90 or above' (C6). (BCI means bridge condition index; a score of 90 or above indicates the structure is in very good condition (Atkins, 2007; Sterritt and Shetty, 2002).) Another interviewee suggested that their organisation could be interested in using condition metrics in future contracts with service providers to monitor performance.

\section{Taking management decisions}

Some common themes were identified across the organisations studied with respect to management decisions (despite variability in systems and processes): (a) prioritisation processes, (b) lifecycle planning, (c) standard asset operating policies and $(d)$ engineering judgement.

\subsection{Prioritisation processes}

For some organisations, prioritisation of work is quite a simple process: 'the priority is often very simple... we've a high, medium or low priority' (C5), while other bodies adopt quantitative approaches: 'we've got our own priority scoring system ... which relates to the importance of the element, the severity of the defect, the size of the structure in terms of deck area, and cost' (C4). One interviewee (C7) described the process of prioritisation as

we have an inspection programme, which highlights defects in structures, which generates what we call a risk score ... those highest risk scores go forward to a renewals programme and what we then try and do is, through value management, prioritise those renewals. (C7)

At least two interviewees referred to a 'value management' process in their organisations but there were differences in whether such processes are used to prioritise interventions or study options. Not all organisations incorporated cost at this stage. Some attempted to determine what may be termed the 'ratio of risk reduction per pound of spend': 'effectively, we start off with the three risk categories and then we prioritise on that, and then we... put the costs against each of those items there, and then we get a value ratio' (C2). Another approach involves estimating the ratio of future expected savings in terms of whole-life cost divided by immediate cost analysed in conjunction with risk scores.

Some of the owners had an aspiration for computer systems to combine deterioration modelling and condition monitoring to automatically produce a prioritised maintenance plan, but there was scepticism as to whether it is possible at stock level with current technology maturity: 'I don't believe we've got a level of sophistication within that arena to begin to determine what your prioritised programme of interventions are' $(\mathrm{C} 15)$. However, such systems were reported to be in place for one flagship project on an individual structure, with one interviewee (C14) saying

They tend to be slightly bespoke, but there are decision support tools stuck to certain structures ... the decision support tool [for one strategically important structure] looks at extrapolated condition as one thing, but it's only one of many factors. Other important things are the consequence of loss of an asset, the traffic management requirements and the benefits of combining with other work. The deterioration curves are used initially to get the initial plan, but then you should get the data to update it ... of course if you've got data on corrosion, carbonation, cracking/spalling and you've got a couple of snapshots in time you 
Table 4. Summary of key findings

\begin{tabular}{ll} 
Finding & Summary of support \\
\hline $\begin{array}{l}\text { Most organisations remain reliant on visual inspections to } \\
\text { monitor the condition of their bridges }\end{array}$ & All interviewee responses supported this conclusion \\
The use of SHM is limited, except for targeted & No current monitoring reported (C3, C4 and C6) \\
monitoring where there are known issues & Examples of targeted monitoring on a small number of specific structures (C1, \\
& C2, C5, C7, C11, C12 and C15) \\
& Significant monitoring systems on a large portion of the assets managed (C8, \\
& C9 and C10) (it is notable that each of these interviewees are responsible for \\
& small numbers of complex assets)
\end{tabular}

can fit deterioration curves and then update them with actual data. Again, this is in pockets, I'm not pretending we're doing this across the board. (C14)

\subsection{Lifecycle planning}

Consideration of the asset lifecycle was common in the surveyed set. For instance, modelling of deterioration effects as well as estimates of whole-life cost are used to plan 'preventative maintenance' efforts: '[the system] tries to predict the condition of different elements over the next 30/40 years, which gives us an indication of ... we don't have to do that now, we can do that in 5 years' time etcetera' $(\mathrm{C} 4)$. One of the surveyed organisations possesses the capability to review the costs and effects of different maintenance strategies for their whole asset stock: 'the whole-life-costing is based on our lifecycle plans ... in terms of putting the programme together as a whole... we will also do an absolute minimum scenario, see what does that look like, we'll run an optimised programme, what does that look like' (C2). Some interviewees reported frustrated attempts to adopt decision support tools based on whole-life costing, with one interviewee (C5) stating

We've tried in the past ... we used to have a system [which] I never got on with because it always came up with the same answer in my mind, which was 'the cheapest option today is the best'. (C5)

\subsection{Standard asset operating policies}

An asset manager may specify standard operating policies for various types of assets and components. Such a policy may require standard interventions for commonly identified defects as well as prescribing 'trigger levels' (i.e. threshold checks (cf. Webb et al., 2015)) that, when reached (or exceeded), certain types of intervention are undertaken. Such efforts may assist the transition from reactive maintenance to preventative maintenance. As noted by one interviewee: 'so those maintenance manuals will have "this area once every $\mathrm{x}$ years" so there's a rolling programme you take out every year' (C8). However, some participants were more sceptical about prescribed maintenance based on standard policies: 'you can make some broad assumptions about deteriorations but you've always got to look at the particular condition of those assets' $(\mathrm{C} 1)$.

\subsection{Engineering judgement}

Those interviewed emphasised that 'engineering judgement' remains critical for decision-making: 'engineering judgement still rules the day' (C5). This chimes with the statement of Shepard (2005: p164). that the 'appropriate use of technology and a modern BMS [bridge management system] should be used strictly as decision support tools - not as a replacement of engineering judgement'. The importance of peer review was also mentioned: 'we have a peer review process to evaluate decisions ... where I have to pitch to my peers' (C5). When discussing the work to be done with a client: 'the list I produce gets discussed at the monthly meetings, so it's pretty much pencilled in at that point which [schemes] are going to be focused on' (C11).

\section{Conclusions}

The key findings from this study are summarised in (Table 4). Most of the bridge engineers and managers represented in this survey (and the organisations they represent) remain heavily reliant on visual inspections to monitor the condition of their bridge assets to plan maintenance activities. Use of SHM is limited in practice, with the notable exception of targeted cases (i.e. where the issue to be investigated was already known). The evidence collected from the semi-structured interviews gives weight to the observation that significant variation exists between the uses of visual inspection data by the industry. It is acknowledged that a larger sample size may reveal more complex and differing trends than those drawn out in this paper. However, the highlights from the interviews presented in this paper and the developed HPM trees do, in the view of the authors, provide useful insight into the state of bridge asset management practices in the UK.

Although high-level inspection standards and guidance such as BD 63/17 (HE, 2017) and the Inspection Manual for Highway Structures (HA, 2007) exist, the interviews indicated little sharing or standardisation of the inspection data interpretation processes that are used to inform decisions. This suggests that 
asset owners incur extra costs through the need to develop bespoke processes and to validate their outputs. Many interviewees recognised the potential of new 'smart' technologies for improving the efficiency and effectiveness of bridge inspections and data collection. However, none of the interviewees pointed to a current unifying paradigm for implementing such technologies. This suggests that considerable effort is still required to formulate and implement such a paradigm that would underpin the development of a new marketplace for bridge monitoring and inspection services, founded on the emerging technologies, and delivering more cost effective and reliable solutions to asset owners.

\section{Acknowledgements}

The first author was supported by the Engineering and Physical Sciences Research Council (EPSRC) funded Industrial Doctorate Centre: Systems (grant no. EP/G037353/1) and WSP UK Ltd. The authors express their thanks to the individuals who gave their time to participate in this research.

Data Availability Statement: The relevant quotes taken from the interviews are included in this paper after transcription from the recording of each interview. To maintain the anonymity of the interviewed participants, the audio recordings or transcripts cannot be made available in a data repository.

\section{REFERENCES}

Atkins (2007) Guidance Document for Performance Measurement of Highway Structures. Part B1: Condition Performance Indicator. Atkins, London, UK. See https://adeptnet.org.uk/system/files/ documents/Guidance $\% 20$ Document $\% 20$ for $\% 20$ Performance $\%$ 20Measurement- $\% 20$ Part $\% 20 \mathrm{~B} 1 \% 20$ Condition $\% 20$ PI.pdf (accessed 03/07/2019).

Bennetts J (2019) The Management of Bridges. EngD thesis, University of Bristol, Bristol, UK

Bennetts J, Vardanega PJ, Taylor C and Denton S (2016) Bridge data what do we collect and how do we use it? In Transforming the Future of Infrastructure Through Smarter Information: Proceedings of the International Conference on Smart Infrastructure and Construction (Mair RJ, Soga K, Jin Y, Parlikad AK and Schooling JM (eds)). ICE Publishing, London, UK, pp. 531-536.

Bennetts J, Webb G, Denton S, Vardanega PJ and Loudon N (2018a) Quantifying uncertainty in visual inspection data. In Maintenance, Safety, Risk, Management and Life-Cycle Performance of Bridges: Proceedings of the 9th International Conference on Bridge Maintenance, Safety and Management (IABMAS 2018) (Powers N, Frangopol D, Al-Mahaidi R and Capriani C (eds)). Taylor \& Francis, London, UK, pp. 2252-2259.

Bennetts J, Webb GT, Vardanega PJ, Denton SR and Loudon N (2018b) Using data to understand trends in bridge performance. Proceedings of the Institution of Civil Engineers - Smart Infrastructure and Construction 171(1): 14-28, https://doi.org/ 10.1680/jsmic.17.00022.

Blockley D and Godfrey P (2000) Doing it Differently. Thomas Telford, London, UK.

Blockley D, Agarwal J and Godfrey P (2012) Infrastructure resilience for high-impact low-chance risks. Proceedings of the Institution of
Civil Engineers - Civil Engineering 165(6): 13-19, https://doi.org/ 10.1680/cien.11.00046.

Davila Delgado JM, Butler LJ, Gibbons N et al. (2017) Management of structural monitoring data of bridges using BIM. Proceedings of the Institution of Civil Engineers - Bridge Engineering 170(3): 204-218, https://doi.org/10.1680/jbren.16.00013.

Davis JP and Hall JW (2003) A software-supported process for assembling evidence and handling uncertainty in decision-making. Decision Support Systems 35(3): 415-433, https://doi.org/10. 1016/S0167-9236(02)00117-3.

Davis J, MacDonald A and White L (2010) Problem-structuring methods and project management: an example of stakeholder involvement using hierarchical process modelling methodology. Journal of the Operational Research Society 61(6): 893-904, https://doi.org/ 10.1057/jors.2010.12.

Flaig KD and Lark RJ (2000) The development of the UK bridge management systems. Proceedings of the Institution of Civil Engineers - Transport 141(2): 99-106, https://doi.org/10.1680/ tran.2000.141.2.99.

Glaser B and Strauss A (1967) The Discovery of Grounded Theory. Aldine, Chicago, IL, USA.

Graybeal B, Phares B, Rolander D, Moore M and Washer G (2002) Visual inspection of highway bridges. Journal of Nondestructive Evaluation 21(3): 67-83, https://doi.org/10.1023/A:1022508121821.

HA (Highways Agency) (2007) Inspection Manual for Highway Structures. Volume 1: Reference Manual. The Stationary Office, London, UK.

Hall JW, Le Masurier JW, Baker-Langman EA, Davis JP and Taylor CA (2004) A decision-support methodology for performance-based asset management. Civil Engineering and Environmental Systems 21(1): 51-75, https://doi.org/10.1080/1028660031000135086.

HE (Highways England) (2017) Design Manual for Roads and Bridges. Volume 3 Highway structures: Inspection and Maintenance. BD 63/17 Inspection of Highway Structures. HMSO, London, UK. See http://www.standardsforhighways.co. uk/ha/standards/dmrb/vol3/section1/bd6317.pdf (accessed 03/07/2019).

Hoult N, Bennett PJ, Stoianov I et al. (2009) Wireless sensor networks: creating 'smart infrastructure'. Proceedings of the Institution of Civil Engineers - Civil Engineering 162(3): 136-143, https://doi. org/10.1680/cien.2009.162.3.136.

Kemmis S, McTaggart R and Nixon R (2014) The Action Research Planner: Doing Critical Participatory Action Research. Springer, Singapore, Singapore.

Lane DC (1992) Modelling as learning - a consultancy methodology for enhancing learning in management teams. European Journal of Operational Research 59(1): 64-84, https://doi.org/10.1016/ 0377-2217(92)90007-V.

Lea F and Middleton C (2002) Reliability of Visual Inspection of Highway Bridges. Department of Engineering, University of Cambridge, Cambridge, UK, Technical Report CUED/ D-STRUCT/TR.201.

McRobbie SG, Wright MA and Chan A (2015) Can technology improve routine visual bridge inspections? Proceedings of the Institution of Civil Engineers - Bridge Engineering 168(3): 197-207, https://doi.org/10.1680/jbren.12.00022.

Middleton C (2004) Bridge management and assessment in the UK. In Proceedings of the Austroads 2004 Bridge Conference: Bridges Another Dimension. Austroads, Sydney, NSW, Australia. See http://www.bridgeforum.org/files/pub/2004/austroads5/Middleton $\%$ 20keynote.pdf (accessed 03/07/2019).

Middleton CR, Fidler PRA and Vardanega PJ (2016) Bridge Monitoring A Practical Guide. ICE Publishing, London, UK. 
Moore M, Phares B, Graybeal B, Rolander D and Washer G (2001) Reliability of Visual Inspection for Highway Bridges, Volume 1. US DOT, Federal Highway Administration, Washington, DC, USA.

NR (Network Rail) (2017) NR/L3/CIVI006: Handbook for the Examination of Structures. Network Rail, London, UK.

Oppenheim AN (1992) Questionnaire Design, Interviewing and Attitude Measurement, new edn. Pinter, London, UK.

Saunders M, Lewis P and Thornhill A (2009) Analysing qualitative data. In Research Methods for Business Students, 6th edn, Pearson Education, Harlow, UK, pp. 544-594.

Shepard RW (2005) Bridge management issues in a large agency. Structure and Infrastructure Engineering 1(2): 159-164, https://doi.org/10.1080/15732470412331289378.
Sterritt G and Shetty N (2002) Bridge Condition Indicators, Volume 1. WS Atkins for CSS Bridges Group, London, UK.

Thurlby R (2013) Managing the asset time bomb: a system dynamics approach. Proceedings of the Institution of Civil Engineers -

Forensic Engineering 166(3): 134-142, https://doi.org/10.1680/feng. 12.00026.

Webb GT, Vardanega PJ, Fidler PRA and Middleton CR (2014) Analysis of structural health monitoring data from Hammersmith flyover. Journal of Bridge Engineering, ASCE 19(6): 05014003, https://doi.org/10.1061/(ASCE)BE.1943-5592.0000587.

Webb GT, Vardanega PJ and Middleton CR (2015) Categories of SHM deployments: technologies and capabilities. Journal of Bridge Engineering, ASCE 20(11): 04014118, https://doi.org/10.1061/ (ASCE)BE.1943-5592.0000735.

\section{How can you contribute?}

To discuss this paper, please email up to 500 words to the editor at journals@ice.org.uk. Your contribution will be forwarded to the author(s) for a reply and, if considered appropriate by the editorial board, it will be published as discussion in a future issue of the journal.

Proceedings journals rely entirely on contributions from the civil engineering profession (and allied disciplines). Information about how to submit your paper online is available at www.icevirtuallibrary.com/page/authors, where you will also find detailed author guidelines. 